CLINICAL STUDY

\title{
Is systematic screening for thyroid disorders indicated in subfertile men?
}

\author{
K Poppe, D Glinoer ${ }^{2}$, H Tournaye ${ }^{1}$, U Maniewski, P Haentjens and B Velkeniers \\ Department of Endocrinology and ${ }^{1}$ Centre for Reproductive Medicine, Vrije Universiteit Brussel (AZ-VUB) Laarbeeklaan 101, 1090 Brussels, Belgium \\ and ${ }^{2}$ Université Libre de Bruxelles, CHU Saint-Pierre, Hoogstraat, 322, B-1000 Brussels, Belgium \\ (Correspondence should be addressed to K Poppe; Email: hemopek@az.vub.ac.be)
}

\begin{abstract}
Context: Data on the prevalence of thyroid disorders in male subfertility remain scarce.

Objective: To investigate the prevalence of thyroid dysfunction and thyroid autoimmunity in men with normal and abnormal semen characteristics.

Setting: Tertiary referral center for reproductive medicine of the University Hospital AZ-VUB, Brussels, Belgium.

Patients and design: Two hundred and ninety-two men were stratified according to the presence of normal (group 1; $n=39$ ) or abnormal (group 2;n=253) semen characteristics. Thyroid function was assessed by serum thyrotropin (TSH) and free thyroxine (FT4), and thyroid peroxidase antibodies (TPO-Ab) for thyroid autoimmunity (TAI or TPO-Ab $>34 \mathrm{kU} / \mathrm{l}$ ); both were correlated with semen characteristics.

Main outcome measures: Semen characteristics were determined by World Health Organisation criteria (rapid + slow motility $\geq 50 \%$ and concentration $\geq 20 \times 10^{6}$ ) and Kruger criteria (morphology $\geq 14 \%$ normal cells).

Results: In group 1 , the mean ( \pm S.D.) age was $33 \pm 4$ years; serum TSH was $1.6(0.3-29.6) \mathrm{mU} / \mathrm{l}$ (median (range)) and FT4 was $12.2(8.8-15.6) \mathrm{ng} / \mathrm{l}$. In group 2, the mean age was $33 \pm 5$ years, serum TSH was $1.3(0.3-5.2) \mathrm{mU} / \mathrm{l}$ and FT4 was $12.5(8.4-17.5) \mathrm{ng} / \mathrm{l}$; (compared with group $1 \mathrm{P}=$ 0.008 for TSH and $P=0.037$ for FT4). In both groups, one patient had increased TSH $(2.6 \%$ and $0.4 \% ; P=$ not significant (ns)). In group 1, one patient had TAI and in group 2 twelve patients had TAI $(2.6 \%$ compared with $4.7 \%$; $=$ ns). FT4 was an independent determinant for semen characteristics.

Conclusions: The prevalence of thyroid dysfunction and autoimmunity is comparable between men with normal and abnormal semen characteristics. On the basis of these data, we do not advise systematic screening for thyroid disorders in subfertile men consulting a tertiary referral center for reproductive medicine.
\end{abstract}

European Journal of Endocrinology $154363-366$

\section{Introduction}

Infertility, defined as the absence of pregnancy within one year of regular unprotected intercourse, is present in $10-15 \%$ of couples in developed countries. The usual causes of infertility include a male factor $(30 \%)$, a female factor (35\%), a combination of both $(20 \%)$, and finally unexplained or 'idiopathic' infertility (15\%) (1). Although thyroid disorders (i.e. hyperthyroidism, hypothyroidism and thyroid autoimmunity or TAI) are less frequent in men than in women, they remain relatively common diseases. The prevalence of TAI in men of reproductive age $(20-40$ years) is $7 \%$ for thyroid peroxidase antibodies (TPO-Ab) and 5\% for thyroglobulin antibodies. Increased serum thyrotropin ( $\mathrm{TSH}>4.5 \mathrm{mU} / \mathrm{l})$ is present in $3.4 \%$ of the men and decreased TSH $(<0.4 \mathrm{mU} / \mathrm{l})$ in $1.8 \%$ of the men in the USA (2). However, in areas with a lower iodine intake (such as Belgium), the prevalence of TAI and supranormal serum TSH may be different $(3,4)$. Associations between thyroid disorders and female infertility have been more extensively studied than associations between thyroid disorders and male infertility $(5,6)$. Thyroid dysfunction can impair the quality of semen, and lower sperm motility and/or concentration. Furthermore, an association between TAI and asthenozoospermia has been described. Semen alterations were reversible after patients 
achieved euthyroidism. The impact of thyroid (dys)function on the semen is mainly due to an effect on central (luteinizing hormone/follicle-stimulating hormone) and peripheral gonadal function and via sex hormone binding proteins (for review see Krassas \& Pontikides (7)).

The aim of this study was to assess whether thyroid dysfunction and/or TAI were more frequently present in men of subfertile couples with normal semen compared with men with altered semen characteristics and whether systematic screening should be undertaken in a tertiary referral center for reproductive medicine.

\section{Materials and methods}

\section{Subjects}

A prospective study was undertaken in 292 men of subfertile couples consulting at the Centre for Reproductive Medicine between October 1999 and November 2000 (tertiary referral center). After informed consent, all men were systematically screened for thyroid function by means of serum TSH and free thyroxine (FT4), and for the presence of TAI with TPO-Ab, before assisted reproductive technology (ART). Men with active thyroid disorders (taking thyroxine, antithyroidea (i.e. propylthiouracyl and/or methimazole) or having an obvious clinical goiter) were excluded from the study. Furthermore, semen analysis was performed and the presence of sperm antibodies was established.

\section{Methods}

Serum assay TSH and FT4 were measured using a third generation electro-chemiluminescence immunoassay (Roche, Mannheim, Germany). The reference values were 0.27 to $4.2 \mathrm{mU} / \mathrm{l}$ for $\mathrm{TSH}$ and 9.3 to $18.0 \mathrm{ng} / \mathrm{l}$ (12 to $23.2 \mathrm{pmol} / \mathrm{l}$ ) for FT4 (conversion factor for FT4 $(\mathrm{ng} / \mathrm{l} \rightarrow \mathrm{pmol} / \mathrm{l})$ : 1.29). TPO-Ab were determined using an RIA kit (B.R.A.H.M.S. Diagnostica, Berlin, Germany). The reference range was $0-34 \mathrm{kU} / \mathrm{l}$. TPO-Ab titers were considered positive when they exceeded $34 \mathrm{kU} / \mathrm{l}$.
Semen analysis Semen was obtained after 3 to 5 days of abstinence, collected in a sterile cup by masturbation at home or in the laboratory and was always analyzed within one hour and at body temperature. If there was any doubt about the semen quality, a second sample was obtained. Liquefaction, appearance, viscosity and volume were evaluated according to World Health Organisation (WHO) guidelines (8). Also, motility and semen concentration were examined according to WHO criteria, but for morphology the Kruger criteria were used (9). Sperm antibodies were detected by a direct mixed antiglobulin reaction test (the MAR test; FertiPro, Ghent, Belgium). After fresh untreated sperm were mixed with latex particles coated with human IgG (or IgA), a mixture of monospecific antihuman IgG (or IgA) antiserum was added. The formation of agglutinates indicated the presence of antibodies and the test was considered positive when $\geq 50 \%$ of the spermatozoa had latex particles attached. Men with azoospermia were excluded from the study.

\section{Statistical analysis}

The classified variables (TPO-Ab) were analyzed by means of a Fisher's exact test; differences between mean values (age) were analyzed by a Student's t-test and median values (TSH and FT4) by the Mann-Whitney U-test. The effects of explanatory variables in men with abnormal semen were analyzed by multiple linear regression. All data analyses were performed using SPSS version 12.0 (SPSS, Inc., Chicago, IL, USA).

\section{Results}

Table 1 shows thyroid function tests (expressed as median (range)) and the number of patients with TAI (measured by TPO-Ab) in all men and in the subgroups. Group 1 (normal semen) comprised 39 men and group 2 (abnormal semen) comprised 253 men. In the entire study group $(n=292)$, the men's mean age (mean \pm S.D.) was $33 \pm 5$ years (range: 20-50 years); mean ages were comparable between the groups.

None of the men in either study group had a suppressed serum TSH concentration. Median (range) serum TSH was slightly but significantly higher in

Table 1 Thyroid function and autoimmunity in all men, and according to the semen analysis.

\begin{tabular}{lccc}
\hline & All men $(n=292)$ & Group 1 normal semen $(n=39)$ & Group 2 altered semen $(n=253)$ \\
\hline Age (years) & $33 \pm 5$ & $33 \pm 4$ & $33 \pm 5$ \\
TSH (mU/l) Reference values: $0.27-4.2$ & $1.6(0.3-29.6)$ & $1.6(0.3-29.6)^{*}$ & $1.3(0.3-5.2)$ \\
FT4 $(\mathrm{ng} / \mathrm{l})$ Reference values: $9.3-18.0$ & $12.5(8.4-17.5)$ & $12.2(8.8-15.6)^{*}$ & $1(2.6 \%)$ \\
TPO-Ab\# & $13(4.5 \%)$ & $12.5(8.4-17.5)$ & $12(4.7 \%)$ \\
\hline
\end{tabular}

Numbers in parentheses represent ranges.

\# Number (\%) of men with TPO-Ab > $34 \mathrm{kU} / \mathrm{l}$.

${ }^{*} P<0.05$ compared with group 2 . 
group 1 compared with group 2 (1.6 (0.3-29.6) compared with $1.3(0.3-5.2) ; P=0.008)$. In both groups one patient had an increased serum TSH level (respectively $2.6 \%$ in group 1 and $0.4 \%$ in group 2; $P=$ not significant (ns)). Median FT4 was significantly lower in group 1 compared with group 2 (12.2 (8.815.6) compared with $12.5(8.4-17.5)$; $P=0.037)$. In group 1 , one patient had an infranormal FT4 (with associated TAI) and in group 2 four patients had an infranormal FT4 (none with TAI); respectively $2.6 \%$ vs $1.6 \% ; P=$ ns. The percentage of TAI in group 1 was comparable to that in group $2(2.6 \%$ vs $4.7 \% ; P=\mathrm{ns})$.

In Table 2 semen abnormalities are described. The majority of the men had teratozoospermia $(n=108$ or $43 \%$ ) and a combination of oligo-astheno-teratozoospermia ( $n=89$ or $35 \%$ ).

In 255 men of the total cohort, the MAR test was performed. In group 1, no man had a positive test compared with 9 in group $2(0 \%$ vs $3.7 \% ; P=n s)$. None of the men with a positive MAR test had positive TPO-Ab. Multiple linear regression revealed that FT4 was a significant predictor for abnormal semen $(P=0.03)$.

\section{Discussion}

The aim of the present study was to investigate whether thyroid dysfunction and/or thyroid autoimmunity were more frequently present in men of subfertile couples with normal semen, compared with those with altered semen characteristics. This could answer the question whether systematic screening for thyroid disorders is indicated in a tertiary referral infertility center. Previous studies mainly investigated the impact of thyroid disorders and/or TAI on semen characteristics.

In the present study, there was no single man with a suppressed serum TSH, highlighting the rarity of the association because of the low prevalence of hyperthyroidism in the male population $(2-4)$. The association between hyperthyroidism and spermatogenesis is poorly covered in the literature, with only two articles published in the 1970s and three during the last decade (10-14). Low sperm densities, lower motility and decreased sperm counts have been reported, although these changes were not more frequently present compared with euthyroid controls (however, only a small number of patients were included). Semen parameters such as fructose, zinc and mercury remained comparable between hyper- and euthyroid men. These

Table 2 Descriptive analysis of sperm abnormalities.

\begin{tabular}{llllllll}
\hline & $\mathbf{O}$ & $\mathbf{A}$ & $\mathbf{T}$ & $\mathbf{A}+\mathbf{T}$ & $\mathbf{O}+\mathbf{A}$ & $\mathbf{0}+\mathbf{T}$ & $\mathbf{O}+\mathbf{A}+\mathbf{T}$ \\
\hline$n$ & 4 & 7 & 108 & 29 & 3 & 13 & 89 \\
$\%$ & 1.5 & 2.7 & 42.6 & 11.5 & 1.2 & 5.1 & 35.2 \\
\hline
\end{tabular}

O, oligospermia; $A$, asthenospermia; $\mathrm{T}$, teratozoospermia. changes were reversed soon after the patients were treated for their hyperthyroidism (14). Together, these observations suggest that hyperthyroidism may impair spermatogenesis.

The number of patients with overt hypothyroidism in the present study was comparable in both study groups, although the median serum FT4 was slightly but significantly higher in men with abnormal sperm. Moreover, the median levels of FT4 remained in the reference range and the differences seem too subtle to explain changes in sperm characteristics. The percentage of hypothyroidism in the present study is also comparable to that in the male population of reproductive age $(2-4)$. Increased serum TSH was present in one man in both study groups. The significantly higher median serum TSH in group 1 was probably due to one outlier (TSH $>29 \mathrm{mU} / \mathrm{l})$. The association between hypothyroidism and spermatogenesis has already been studied thirty years ago in two clinical studies showing decreased seminal volume, motility and abnormal testicular biopsies. These studies included a small number of patients and often lacked a control population $(15,16)$. More recent studies followed a number of postpubertal men after hypothyroidism was induced for three months. Moderate semen abnormalities, such as decreased sperm volume and forward motility were observed, insufficient however to cause infertility (17). Other studies showed a decreased seminal volume, sperm motility and abnormal testicular biopsies. After treatment with levothyroxine, the semen parameters tended to normalize $(17,18)$.

In the present study, the prevalence of TAI was almost doubled in men with abnormal semen compared with that in men with normal semen, although this was not statistically significant. This could be due to the small number of patients with TAI in group 1 $(n=1)$ or a beta error. Furthermore, no correlation between the MAR test and TAI could be detected. The association of TAI and sperm antibodies has been investigated in two studies. An increased incidence of thyroid antibodies in men with sperm autoantibodies compared with men without sperm antibodies was shown independent from a history of genital tract obstruction. These studies postulated that TAI and other organspecific antibodies were probably related to a common immune dysregulation (19, 20). More recently, Trummer et al. (21) investigated prospectively the impact of thyroid dysfunction and autoimmunity on semen in a large cohort of subfertile men. In the latter group, TAI was found in $7.5 \%$ of the men and elevated thyroid antibody titers were significantly correlated with asthenozoospermia. Subclinical hypothyroidism was present in 3\% of the cases, but was not correlated with altered semen density, sperm motility or morphology (21).

In conclusion: although in the literature some evidence exists that thyroid disorders and thyroid autoimmunity can influence sperm parameters (as a 
surrogate endpoint of male infertility), no increased prevalence of thyroid disease was detected in a large cohort of men with altered semen compared with men with normal semen. Therefore, there does not seem to be any justification to recommend systematic screening for thyroid function and thyroid autoimmunity in men of subfertile couples consulting a tertiary referral center.

\section{Acknowledgements}

This study was supported by grants from the Willy Gepts Foundation AZ-VUB; Daniel Glinoer participated to the study within the framework of Contract ARC $\mathrm{N}^{\circ}$ 04/09-314.

\section{References}

1 Hoxsey R \& Rinehart JS. Infertility and subsequent pregnancy. Clinics in Perinatology 199724 321-342.

2 Hollowell JG, Staehling NW, Flanders WD, Hannon WH, Gunter EW, Spencer CA \& Braverman LE. Serum TSH, T4, and thyroid antibodies in the United States population (1988 to 1994): National Health and Nutrition Examination Survey (NHANES III). Journal of Clinical Endocrinology and Metabolism $200287489-499$.

3 Bjoro T, Holmen J, Kruger O, Midthjell K, Hunstad K, Schreiner T, Sandnes L \& Brochmann H. Prevalence of thyroid disease, thyroid dysfunction and thyroid peroxidase antibodies in a large, unselected population. The Health Study of Nord-Trondelag (HUNT). European Journal of Endocrinology 2000143 639-647.

4 Knudsen N, Bülow I, Jørgensen T, Laurberg P, Ovesen L \& Perrild H. Comparative study of thyroid function and types of thyroid dysfunction in two areas in Denmark with slightly different iodine status. European Journal of Endocrinology 2002143 485-491.

5 Poppe K, Glinoer D, Van Steirteghem A, Tournaye H, Devroey P, Schiettecatte J \& Velkeniers B. Thyroid dysfunction and autoimmunity in infertile women. Thyroid $200211995-999$.

6 Poppe K \& Velkeniers B. Female infertility and the thyroid. Best Practice and Research, Clinical Endocrinology and Metabolism 2004 18 153-165.

7 Krassas GE \& Pontikides N. Male reproductive function in relation with thyroid alterations. Best Practice and Research, Clinical Endocrinology and Metabolism 200418 183-195.
8 World Health Organization. WHO Laboratory Manual for the Examination of Human Semen and Sperm-Cervical Mucus Interaction (4th ed). Cambridge: Cambridge University Press, 1999.

9 Kruger TF, Acosta AA, Simmons KF, Swanson RJ, Matta JF \& Oehninger S. Predictive value of abnormal sperm morphology in in vitro fertilization. Fertility and Sterility 198849 112-117.

10 Clyde HR, Walsh PC \& English RW. Elevated plasma testosterone and gonadotropin levels in infertile males with hyperthyroidism. Fertility and Sterility $197627662-666$.

11 Kidd GS, Glass AR \& Vigersky RA. The hypothalamic-pituitarytesticular axis in thyrotoxicosis. Journal of Clinical Endocrinology and Metabolism 197948 798-802.

12 Hudson RW \& Edwards AL. Testicular function in hyperthyroidism. Journal of Andrology 199213 117-124.

13 Abalovich M, Levalle O, Hermes R, Scaglia H, Aranda C, Zylbersztein C, Oneto A, Aquilano D \& Gutierrez S. Hypothalamic-pituitary-testicular axis and seminal parameters in hyperthyroid males. Thyroid $19999857-863$.

14 Krassas GE, Pontikides N, Deligianni V \& Miras K. A prospective controlled study of the impact of hyperthyroidism on reproductive function in males. Journal of Clinical Endocrinology and Metabolism 200287 3667-3671.

15 Griboff SI. Semen analysis in myxedema. Fertility and Sterility $196213436-443$.

16 De La Balze FA, Arrilla F, Mancini RE, Janches M, Davidson OW \& Gurtman AI. Male hypogonadism in hypothyroidism: a study of six cases. Journal of Clinical Endocrinology and Metabolism 1962 22 212-222.

17 Corrales Hernandez JJ, Miralles Garcia JM \& Garcia Diez LC. Primary hypothyroidism and human spermatogenesis. Archives of Andrology 199025 21-27.

18 Jaya Kumar B, Khurana ML, Ammini AC, Karmarkar MG \& Ahuja MM. Reproductive endocrine functions in men with primary hypothyroidism: effect of thyroxine replacement. Hormone Research 199034 215-218.

19 Baker HW, Clarke GN, McGowan MP, Koh SH \& Cauchi MN. Increased frequency of autoantibodies in men with sperm antibodies. Fertility and Sterility $1985 \mathbf{4 3} 438-441$.

20 Paschke R, Bertelsbeck DS, Tsalimalma K \& Nieschlag E. Association of sperm antibodies with other autoantibodies in infertile men. American Journal of Reproductive Immunology 199432 $88-94$.

21 Trummer H, Ramschak-Schwarzer S, Haas J, Habermann H, Pummer K \& Leb G. Thyroid hormones and thyroid antibodies in infertile males. Fertility and Sterility 200176 254-257.

Received 23 September 2005

Accepted 23 November 2005 